\title{
Closed Orbit Correction Using Singular Value Decomposition of the Response Matrix*
}

\author{
Y. Chung, G. Decker, and K. Evans, Jr. \\ Argonne National Laboratory, Argonne, IL 60439
}

\begin{abstract}
A theory of global orbit correction using the technique of singular value decomposition (SVD) of the response matrix and simulation of its application to the Advanced Photon Source (APS) storage ring are presented. The response matrix relates beam motion at the beam position monitor (BPM) locations to changes in corrector magnet strengths. SVD reconfigures the BPMs and correctors into the same number of "transformed" BPMs (t-BPMs) and "transformed" correctors (t-correctors), each t-BPM being coupled to at most one tcorrector and vice versa with associated coupling strength which determines the efficiency of orbit correction. The coefficients of these linear transformations can be used to determine which BPMs and correctors are the most effective. Decoupling the weakly coupled pairs will enhance the overall correction efficiency at the expense of accuracy. The orbit errors at decoupled t-BPMs are conserved and the strengths of decoupled t-correctors can be adjusted appropriately to optimize the actual corrector strengths. This method allows for estimating the limitation on orbit correction with given sets of BPMs and correctors, as well as optimizing the corrector strengths without overloading the corrector magnet power supplies.
\end{abstract}

\section{INTRODUCTION}

The third generation synchrotron light sources, of which the Advanced Photon Source (APS) is one, are characterized by low emittance of the charged particle beams and high brightness of the photon beams radiated from insertion devices. Transverse stability of the particle beams is a crucial element in achieving these goals and the APS will implement extensive beam position feedback systems, which include 320 corrector magnets, 360 positron beam position monitors (BPMs) distributed around the storage ring, miniature BPMs for insertion device beamlines, and photon beam position monitors in the front end of X-ray beamlines.

The beam position feedback systems can largely be divided into the global and local feedback systems according to the extent of correction, and the DC and AC feedback systems according to the bandwidth of correction.

In this work, we will concentrate on the theory of DC global orbit correction and its application to the APS storage ring. We will show that the global response matrix relating the beam motion at selected BPMs and changes in steering corrector strengths can be transformed into a diagonal matrix. The mechanism of this transformation is provided by the technique of singular value decomposition (SVD)[1-3] of matrices. Each diagonal element represents the correction efficiency of an orbit correction channel and the channels are independent of one another. The AC global orbit correction is then equiv llent to a combination of the DC global correction algorithm and multiple non-interacting feedback systems. The analysis of a single-channel feedback system in frequency and time domains is treated in Ref. [4].

\section{THEORY}

Let us consider M BPMs and N correctors used for closed orbit correction in the storage ring. The i-th BPM has beta and phase functions $\left(B_{i}, \psi_{i}\right)$, and similarly, the $j$-th corrector has $\left(\beta_{c j}, \psi_{c j}\right)$. The response matrix $R_{i j}$ corresponding to the beam motion at the $i$-th BPM per unit angle of kick by the $j$-th corrector is then given by [5]

$$
\mathrm{R}_{i j}=\frac{\sqrt{\beta_{i} \beta_{c i}}}{2 \sin \pi \nu} \cos \left(\left|\psi_{i}-\Psi_{c j}\right|-\pi \nu\right) .
$$

$v$ is the betatron tune of the machine. The response matrix $R_{i j}$ can be obtained from measurements by reading beam position changes while varying the corrector strengths one by one.

\section{A. SVD Formalism}

With the response matrix $\mathbf{R}$ thus obtained, we write $\mathbf{R}$ as a product of three matrices $\mathbf{U}, \mathbf{W}$, and $\mathbf{V}$ as [1]

$$
\mathbf{R}=\mathbf{U} \cdot \mathbf{W} \cdot \mathbf{V} \mathbf{T}
$$

where $U$ is an $M \times M$ unitary matrix $\left(U^{\top} \cdot U=U \cdot U^{\top}=1\right)$, $W$ is an $\mathrm{M} \times \mathrm{N}$ diagonal matrix with positive or zero elements, and $\mathrm{V}$ is an $\mathrm{N} \times \mathrm{N}$ unitary matrix $\left(\mathrm{V}^{\mathrm{T}} \cdot \mathrm{V}=\mathrm{V} \cdot \mathrm{V}^{\mathrm{T}}=1\right) . \mathrm{M}$ is the number of BPMs and $\mathrm{N}$ is the number of correctors. This decomposition is unique only to a certain extent, and there are other ways of decomposing the matrix R. $[2,3]$

Let us denote by $\Delta \mathbf{x}$ the global orbit change due to the corrector strength change $\Delta \theta$ and define

$$
\Delta \mathbf{x}^{\mathrm{i}}=\mathbf{U}^{\mathrm{T}} \cdot \Delta \mathrm{x} \text { and } \Delta \boldsymbol{\theta}^{\mathrm{t}}=\mathrm{V}^{\mathrm{T}} \cdot \Delta \boldsymbol{\theta}
$$

Then, from Eqs. (2) and (3) we have

$$
\Delta \mathbf{x}^{\prime}=\mathbf{W} \cdot \Delta \boldsymbol{\theta}^{\mathfrak{\prime}}
$$

*Work supported by the U.S. Department of Energy, Office of Basic Energy Sciences, under Contract No. W-31-109-ENG-38.

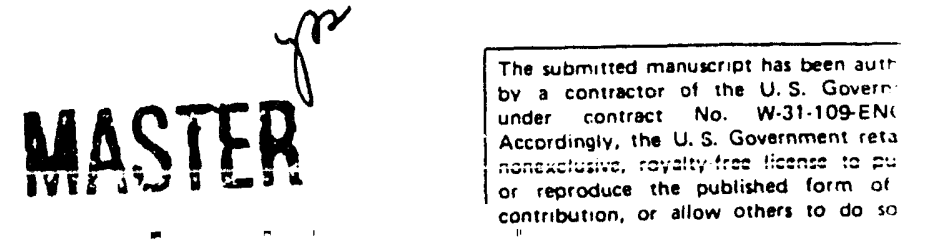


Equation (3) is the rule of transformation for the BPMs and correctors. $\Delta x^{t}$ and $\Delta \theta^{t}$ are the vectors in the transformed BPM (t-BPM) space and transformed corrector (t-corrector) space, respectively. The columns of the matrices $U$ and $V$ are the orthogonal basis vectors $\left\{\mathbf{u}_{\mathrm{i}}\right\}$ and $\left\{\mathbf{v}_{\mathrm{j}}\right\}$. The elements of the matrix $\mathbf{W}$ is given by

$$
W_{i j}=w_{\min (i, j)} \delta_{i j} \text {. }
$$

We call these diagonal elements $w_{n}(\geq 0,1 \leq n \leq \min (M, N))$ eigenvalues, which represent the coupling efficiency between the t-BPMs and t-correctors. The matrix $\mathbf{R}$ is singular if any of the eigenvalues are equal to zero. The basis vectors are related through the relation

$$
\mathbf{R} \cdot \mathbf{v}_{\mathrm{n}}=\mathrm{w}_{\mathrm{n}} \mathbf{u}_{\mathrm{n}} \cdot \quad 1 \leq \mathrm{n} \leq \min (\mathrm{M}, \mathrm{N})
$$

\section{B. Matrix Inversion and Orbit Correction}

Let $\Delta \mathbf{x}$ be the orbit error given by the difference between the reference orbit $x_{\mathrm{r}}$ and the current orbit $\mathbf{x}_{\mathrm{m}}$. That is,

$$
\Delta \mathbf{x}=\mathbf{x}_{\mathrm{r}}-\mathbf{x}_{\mathrm{m}} .
$$

In order to bring the orbit to the reference orbit, we need to calculate $\Delta \theta$ such that

$$
\mathbf{R} \cdot \Delta \boldsymbol{\theta}=\Delta \mathbf{x} .
$$

In case such solutions do not exist, we want the solution that minimizes the difference $|\mathbf{R} \cdot \Delta \boldsymbol{\theta}-\Delta \mathbf{x}|$. SVD provides this solution as

$$
\Delta \boldsymbol{\theta}=\mathbf{R}_{\text {inv }} \cdot \Delta \mathbf{x},
$$

where

$$
\mathbf{R}_{\mathrm{inv}}=\mathbf{V} \cdot \mathbf{W}_{\mathrm{inv}} \cdot \mathbf{U}^{\mathrm{T}} \text {. }
$$

$\mathbf{W}_{\text {inv }}$ is a diagonal matrix of dimension $\mathrm{N} \times \mathrm{M}$ and the elements are given by

$$
W_{i n v, i j}=q_{\min (i, j)} \delta_{i j},
$$

where

$$
q_{n}=\left\{\begin{array}{ll}
0, & w_{n} \leq \varepsilon w_{\max } \\
\frac{1}{w_{n}} & \text { otherwise. }
\end{array} \quad(1 \leq n \leq \min (M, N))\right.
$$

$\varepsilon$ is the singularity rejection parameter in the range $[0,1]$. This parameter is determined primarily by the orbit correction needs and the corrector strength limits. Zero $\mathrm{q}_{\mathrm{n}}$ 's correspond to decoupled channels which do not contribute to orbit correction.

When $\varepsilon=0$, all the non-zero eigenvalues are retained and the most accurate correction will result. However, this will require very robust power supplies for the correctors. On the other hand, if $\varepsilon=1, \mathbf{R}_{\mathrm{inv}}$ is a null matrix and there will be no orbit correction. Usually, $\varepsilon$ is set to the smallest value such that none of the power supplies saturates.

For a given matrix $\mathbf{R}$, we define $\varepsilon_{\mathrm{m}}(\mathbf{R})$ as

$$
\varepsilon_{m}(\mathbf{R})=\max \left\{\varepsilon \mid w_{n}>\varepsilon w_{\text {max }} \text { for all } w_{n} \neq 0\right\} .
$$

That is, $\varepsilon_{\mathrm{m}}$ is the largest possible value for $\varepsilon$ in order to retain all non-zero eigenvalues. The inverse matrix $\mathbf{R}_{\mathrm{inv}}$ satisfies

$\mathbf{R} \cdot \mathbf{R}_{\mathrm{inv}} \cdot \mathbf{R}=\mathbf{R}\left(\varepsilon \leq \varepsilon_{\mathrm{m}}\right)$ and $\mathbf{R}_{\mathrm{inv}} \cdot \mathbf{R} \cdot \mathbf{R}_{\mathrm{inv}}=\mathbf{R}_{\mathrm{inv}}$ (for all $\left.\varepsilon\right)$.

\section{Minimization of Orbit Error}

Orbit correction when the number of BPMs $\mathrm{M}$ is not larger than the number of coupled channels $C(\leq \min (M, N))$ is trivial since the solution that satisfies Eq. (8) always exists. Let us now consider the case when $M$ is larger than $C$, the maximum number that does not saturate the corrector strengths, and let $\Delta \mathbf{x}$ be the initial orbit error. 'Then the new difference orbit $\Delta \mathbf{x}^{\prime}$ after applying the correction $\Delta \boldsymbol{\theta}$ given by Eq. (9), using Eqs. (2) and (3), is

$$
\Delta \mathbf{x}^{\prime}=\left(1-\mathbf{R} \cdot \mathbf{R}_{\mathrm{inv}}\right) \cdot \Delta \mathrm{x}=\mathbf{U} \cdot\left(\mathbf{1}-\mathbf{W} \cdot \mathbf{W}_{\mathrm{inv}}\right) \cdot \mathbf{U}^{\mathrm{T}} \cdot \Delta \mathbf{x},
$$

or, in the t-BPM space,

$$
\Delta \mathbf{x}^{\prime \prime}=\mathbf{U}^{\mathrm{T}} \cdot \Delta \mathbf{x}^{\prime}=\left(1-W \cdot \mathbf{W}_{\mathrm{inv}}\right) \cdot \Delta \mathbf{x}^{\mathrm{t}} \text {. }
$$

Since the transformation conserves vector norm, Eq. (16) gives

$$
\left|\Delta \mathbf{x}^{\prime}\right|=\left|\Delta \mathbf{x}^{\prime}\right|=\left(\sum_{i=c+1}^{M}\left|\Delta \mathbf{x}_{i}^{\prime}\right|^{2}\right)^{1 / 2} .
$$

In Eq. (17), the position error $\Delta \mathrm{x}_{\mathrm{i}}$ is reduced to zero for the coupled $t$-BPMs $(1 \leq \mathrm{i} \leq \mathrm{C})$ after correction, while it is conserved for the decoupled 1 -BPMs $(C+1 \leq \mathrm{i} \leq \mathrm{M})$. Therefore, the orbit error cannot be reduced further than given by Eq. (17) unless $C$ is increased by, e.g., optimizing the corrector strengths. This is also proven by showing that the corrector strengths are not changed any more. From Eq. (14),

$$
\Delta \boldsymbol{\theta}^{\prime}=\mathbf{R}_{\mathrm{inv}} \cdot \Delta \mathbf{x}^{\prime}=\left(\mathbf{R}_{\mathrm{inv}}-\mathbf{R}_{\mathrm{inv}} \cdot \mathbf{R} \cdot \mathbf{R}_{\mathrm{inv}}\right) \cdot \Delta \mathbf{x}=\mathbf{0} .
$$

Particularly, with C equal to N, Eq. (17) is the absolute minimum beyond which no further orbit correction is possible by any method. However, in reality, error in the measurement of the response matrix $\mathbf{R}$, changes in the machine condition, and external perturbations cause residue in the closed orbit error. Correction of this error is done by $\mathrm{AC}$ orbit correction with appropriate bandwidth.

\section{Optimization of Correctors}

When the number of correctors $\mathrm{N}$ is larger than $\mathrm{C}$, the number of coupled channels, the correctors can then be optimized in various ways. Between successive corrections of the closed orbit, some of the correctors can be close to saturation, thus preventing any further corrections. In this 
case, if $\mathrm{N}$ is larger than $\mathrm{C}$, the decoupled t-correctors can be used to relieve those correctors.

Let $\mathbf{V}_{\mathbf{s}}$ be the submatrix of $\mathbf{V}$ that corresponds to the decoupled t-correctors. That is,

$$
\mathrm{V}_{\mathrm{sij}}=\mathrm{V}_{\mathrm{ij}+\mathrm{C} .} .(1 \leq \mathrm{i} \leq \mathrm{N}, 1 \leq \mathrm{j} \leq \mathrm{N}-\mathrm{C})
$$

The desired corrector strengths change, $\Delta \theta_{s}$, is transformed in the subspace of $\mathrm{t}$-correctors spanned by the corrector basis vectors $v_{j}(C+1 \leq j \leq N)$ and then inverse-transformed. The resulting $\Delta \theta_{s}^{\prime}$ given by

$$
\Delta \boldsymbol{\theta}_{\mathrm{s}}{ }^{\prime}=\mathrm{V}_{\mathrm{s}} \cdot \mathbf{V}_{\mathrm{s}}^{\mathrm{T}} \cdot \Delta \boldsymbol{\theta}_{\mathrm{s}}
$$

will then be the closest to the $\Delta \theta_{\mathrm{s}}$ while disturbing the orbit the least. As a special case, when $\Delta \theta_{s}=0$, we have

$$
|\Delta \theta|=\left|\Delta \theta^{\prime}\right|=\left(\sum_{j=1}^{c}\left|\Delta \theta_{j}^{\prime}\right|^{2}\right)^{1 / 2}
$$

since $\Delta \theta_{j}^{t}=0$ for the decoupled $t$-correctors $(C+1 \leq j \leq N)$. That is, $|\Delta \theta|$ given by Eq. (21) is the minimum value possible. Particularly, when $\mathrm{C}=\mathrm{N}$, it is the absolute minimum among all solutions that satisfy Eq. (8).

In a similar manner, if $\boldsymbol{\theta}$ is the current corrector strengths, $\theta$ ' given by

$$
\boldsymbol{\theta}^{\prime}=\mathbf{R}_{\text {inv }} \cdot \mathbf{R} \cdot \boldsymbol{\theta} .
$$

will minimize the overall corrector strengths. In case $\mathrm{N}>\mathrm{C}$, further optimization can be done by applying Eq. (20).

\section{ANALYSIS OF THE APS STORAGE RING}

In this section, we will analyze global orbit correction for the APS storage ring in the vertical plane. There are 40 sectors in the machine and each sector has nine BPMs (total 360) and eight correctors (total 320) available for global orbit correction. The distribution of BPMs and correctors is identical for all sectors.

Figure 1 shows the plot of the BPM basis vectors $U_{i 1}$ and $U_{i 2}$ as functions of the BPM index $i$. These vectors are mutually orthogonal and correspond to the largest eigenvalues $w_{1}$ and $w_{2}$ equal to $1.140 \times 10^{3} \mathrm{~m} / \mathrm{rad}$. They also have the same frequency as the integer tune $\left(v_{V}=14.2987\right)$ of the machine, which means that perturbation with the harmonic number 14 can be corrected the most efficiently. The first two corrector basis vectors $V_{j 1}$ and $V_{j 2}$ show similar behavior.

Figure 2 shows the eigenvalues $w_{n}(1 \leq n \leq 320)$ in descending order when all BPMs and correctors are used. The maximum and minimum values are $1.140 \times 10^{3}$ and $9.126 \times 10^{-2}$ $\left(\varepsilon_{\mathrm{m}}=8.005 \times 10^{-5}\right)$ in units of $\mathrm{m} / \mathrm{rad}$, respectively. The machine periodicity is exhibited in the discontinuous changes of $w_{n}$ at every 40 . The large decrease at $n=240$ indicates that 80 of the correctors are redundant and therefore do not contribute much to orbit correction. These correctors have the smallest values of the function

$$
E(j)=\sum_{n} w_{n} V_{j n}^{2} . \quad(1 \leq j \leq 320)
$$

When those correctors are removed, $\varepsilon_{\mathrm{m}}$ becomes $6.656 \times 10^{-4}$.

The function $E(j)$ is a measure of the efficiency of the $j-$ th corrector. A similar function can be defined for the BPMs and these functions can be used to select a subset of BPMs and correctors with the condition that $\varepsilon_{\mathrm{m}}$ be maximized.

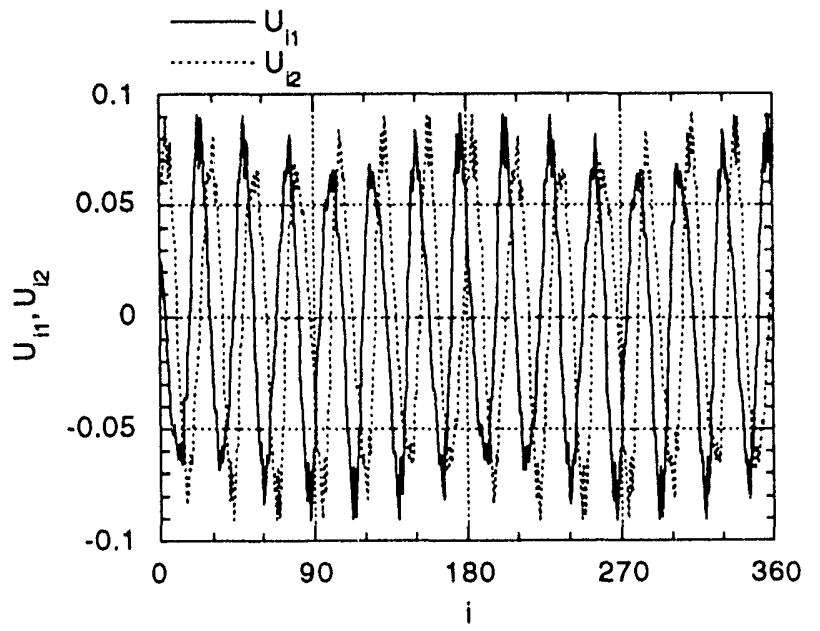

Fig. 1: The BPM basis vectors $U_{i 1}$ and $U_{i 2}(1 \leq i \leq 360)$ for the most strongly coupled channels $\left(w_{1}=w_{2}=1.140 \times 10^{3} \mathrm{~m} / \mathrm{rad}\right)$ in the vertical plane $\left(v_{V}=14.2987\right)$ for the APS storage ring.

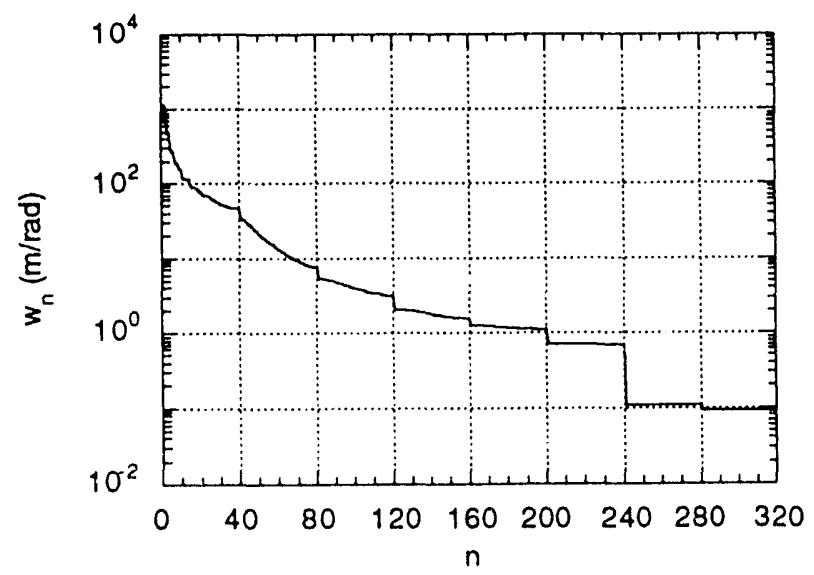

Fig. 2: Plot of the eigenvalues in descending order for the APS sti:age ring. $M=360, N=320 . \varepsilon_{m}=8.005 \times 10^{-5}$.

\section{REFERENCES}

(1] MATLAB ${ }^{\mathrm{TM}}$ User's Guide, The Mathworks, Inc., p. 3-178, 1990.

[2] W. Press et al., Numerical Recipes in $C$, Cambridge University Press, p. 60, 1989.

[3] Mathematica ${ }^{\mathrm{TM}}$, Wolfram Research, Inc., p. 454, 1988.

[4] Y. Chung, L. Emery, and J. Kirchman, "Compensation for the Effect of Vacuum Chamber Eddy Current by Digital Signal Processing for Closed Orbit Feedback," these proceedings.

[5] M. Sands, "The Physics of Electron Storage Rings - An Introduction," SLAC-121, 1970. 
DISCLAIMER

This report was prepared as an account of work sponsored by an agency of the United States Government. Neither the United States Government nor any agency thereof, nor any of their employees, makes any warranty, express or implied, or assumes any legal liability or responsibility for the accuracy, completeness, or usefulness of any information, apparatus, product, or process disclosed, or represents that its use would not infringe privately owned rights. Reference herein to any specific commercial product, process, or service by trade name, trademark, manufacturer, or otherwise does not necessarily constitute or imply its endorsement, recommendation, or favoring by the United States Government or any agency thereof. The views and opinions of authors e:pressed herein do not necessarily state or reflect those of the United States Government or any agency thereof. 

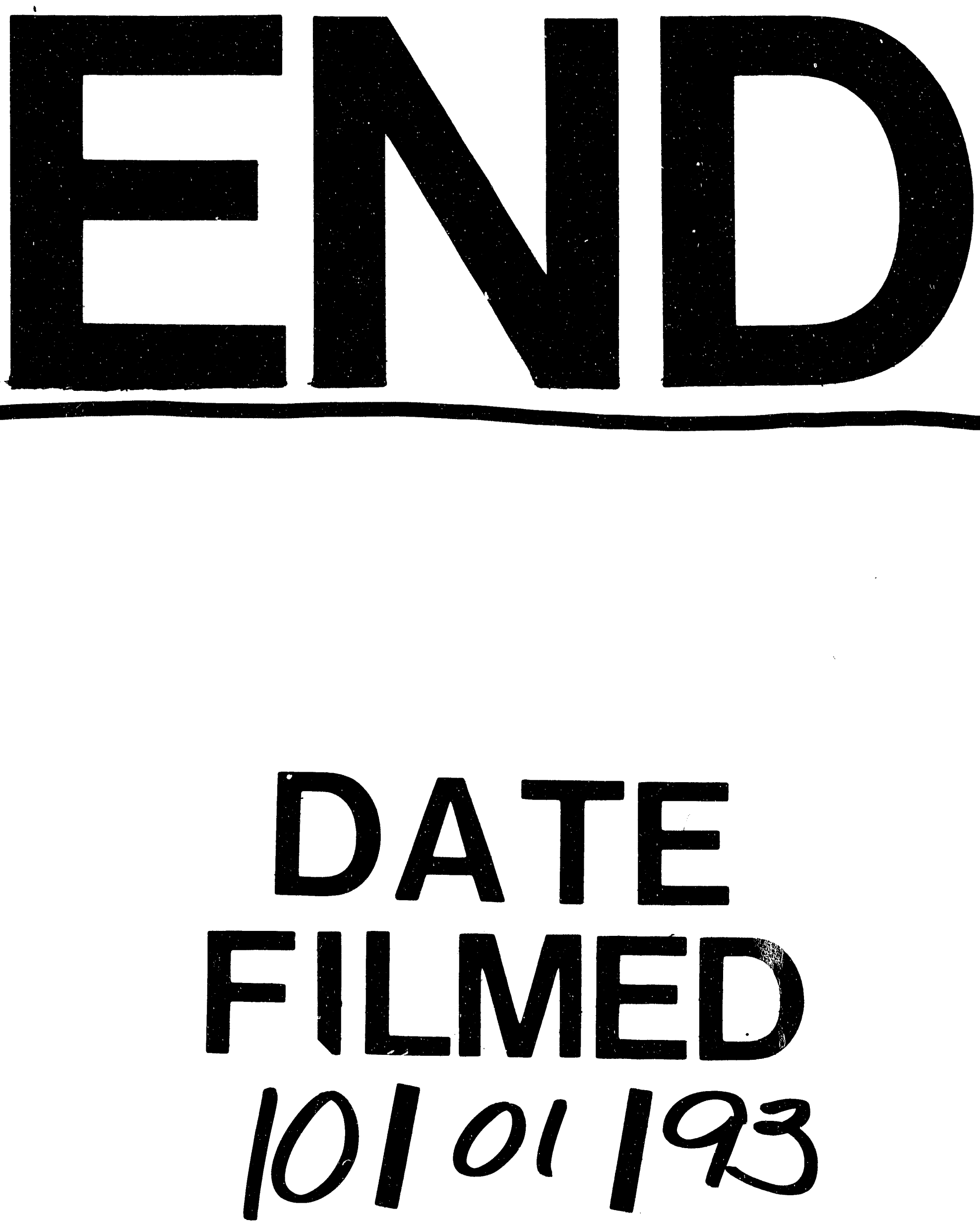
